\title{
Turismo, terrorismo y crisis socio-económica. \\ El caso de Perú (1980-1992)
}

\author{
Tourism, terrorism and socio-economic crisis. \\ The case of Perú (1980-1992)
}

Fernando Armas Asín*

Universidad del Pacífico, Perú

\section{RESUMEN}

En este trabajo se destaca la importancia en distinguir las causas que afectan el turismo en un país que atraviesa situaciones de violencia, crisis económica y la confluencia de otras problemáticas por un periodo largo de tiempo. Se estudia el caso peruano con el objetivo de establecer los elementos más significativos que afectaron el turismo receptivo entre los años 1980 y 1992. En contraposición de lo que comúnmente se sostiene, se plantea que además del terrorismo son las crisis económicas y otros factores sociales y políticos los que deben considerarse para explicar lo acontecido.

Palabras clave: crisis económica, Perú, terrorismo, turismo, violencia.

\begin{abstract}
The article underlines the importance of a proper distinction between the causes that affects tourism in a country that goes though violent situations, economic crisis, and other problems combined, for a long period of time. Through the study of the Peruvian case, the work seeks to establish which elements were the most meaningful in affecting the receptive tourism between 1980 and 1992. Against what is commonly thought, it poses that the economic crisis as well as other social and political factors besides terrorism, must be considered to explain the circumstances.
\end{abstract}

Keywords: economic crisis, Peru, terrorism, tourism, violence. 


\section{Introducción}

Los temas sobre el turismo, la violencia armada y la inestabilidad política, así como sobre el turismo y la crisis económica, han interesado a diversos investigadores. En el primer caso han centrado su atención en los efectos directos en la relación terrorismo y turismo durante las últimas décadas (particularmente luego de los atentados del año 2001en EE.UU.), así como en la violencia política en muchos países considerados destinos turísticos tradicionales (Baumert, 2016, pp. 51-70). En ellos se recuerda que las acciones terroristas contra turistas o lugares de interés suelen ser racionales, tomando en cuenta los países de origen de los turistas o si la actividad es apoyada por los regímenes locales; también, se subraya los estragos que pueden producir con una caída en la cifra de arribos y el cambio de destino de los visitantes, para luego, si no intermedian más factores perturbadores -particularmente políticos o sociales-, empezar un cambio de tendencia, acompañado tal vez de fuertes campañas publicitarias del gobierno, inversión en infraestructura o seguridad. En contraposición las inestabilidades políticas, conflictos internos prolongados, golpes de Estados, entre otros, pueden producir efectos de larga duración en el turismo, más aún si ellos confluyen (Baumert, 2016; Causevic y Lynch, 2013; Drakos y Kutan, 2003; Gonzáles, 2016; Moral, 2016; Saha y Yap, 2013).

Pero si la relación entre turismo y terrorismo ha sido establecida bajo estos marcos, la relación del turismo con las crisis económicas ha merecido menos estudios. Abundan los trabajos monográficos, muchos de ellos modelos econométricos, que establecen los impactos de la crisis de 2008 en una determinada región, el aumento del riesgo, así como también las mejoras, por la fortaleza del turismo interno o por el traslado de flujos receptivos de otra región, entre otros (Aurioles, 2011; Dorta, 2011; Torres, 2014).

Sin embargo, lo que no existen son estudios que planteen la relación dinámica y simultánea entre turismo, crisis económica, crisis política y terrorismo.

Esto es particularmente notorio en América Latina. Los estudios sobre turismo y violencia armada se han centrado en México y Colombia: en el primer caso subrayando el efecto que tuvieron los acontecimientos de 2001 en Estados Unidos para la retracción del turismo norteamericano a México, aunque algunos trabajos considerando la propia inseguridad ciudadana que se vive en el país (Bringas y Verduzco, 2008; Domínguez et al., 2003). En el segundo caso, evaluando la acción de las guerrillas, el narcotráfico y la crisis social en la caída del flujo turístico internacional, como en el riesgo inherente que disuade a muchos turistas a viajar (Fernández, 2002; Gómez, 2016; Jaramillo, 2006; Gómez, 2002; Van Broeck, 2002). Aunque también se ha analizado para Colombia cómo a algunos turistas les interesa el riesgo controlado como una experiencia distinta de sensaciones, de atracción y de identidad (Guilland, 2012). En general, se acepta que no hay un lugar completamente seguro en el planeta (Korstanje, 2013; Korstanje y Tarlow, 2012). El terrorismo, como un fenómeno específico de la violencia, no es una amenaza para el turismo, existiendo una relación compleja entre ambos; de hecho, en algunos países del mundo a veces el turismo crece en coexistencia con este (Korstanje, 2012).

Pero, y a pesar de todos estos desarrollos, no se ha avanzado en la comprensión de largo plazo en otros países de la región con tradición constante de estos fenómenos de violencia y crisis social, y menos estudios que lo combinen con la problemática de las crisis económicas, tomando en cuenta que muchos vivieron la 
combinación de estos fenómenos entre las décadas de 1970 y 1990 en Centroamérica y Sudamérica. De la misma manera, al trabajar la relación entre el turismo y las crisis económicas se las aisló, midiendo mayormente solo sus efectos macroeconómicos, por ejemplo (Morales, 2009). Solo Monti (2011), al estudiar la problemática de la recuperación turística tras una crisis, analizó la importancia de las múltiples políticas en juego. Estas ausencias, en una región con constantes problemas económicos, sociales y políticos, y ahondamiento de las condiciones locales de pobreza, desempleo e informalidad, merecen atención.

América Latina, luego de vivir una etapa intensa de crecimiento del turismo receptivo entre 1950 y 1980 , pasó a una década de oscilaciones en las cifras. En el caso de Brasil, se vio caer su cifra de llegadas de 1.94 millones en 1986 a 1.09 millones en 1990, en un contexto de crisis económica e hiperinflación que lo afectaron a fines de la década. Nicaragua pasó de recibir 288000 turistas en 1980 a contabilizar apenas 49000 en 1987, en un derrumbe que coincide con la aguda crisis económica y la violencia política. Por su parte, Bolivia vio un estancamiento en sus cifras la mayor parte de la década de 1980, en medio de una severa recesión e hiperinflación, notándose solo a partir de 1988 una recuperación. En el caso de Chile, igualmente su crisis alejó a muchos turistas, llegando solo a 362 mil visitantes en 1984, para luego de superar su problemática al alcanzar los 943 000 en 1990. Por las estadísticas se puede inferir su relación con las crisis económicas y la violencia, por lo menos en los países mencionados (Alfaro, 1991, p. 40; CANATUR, 1989, p. 5; Ministerio de Industria, Turismo, Integración y Negociaciones Comerciales Internacionales [MITINCI], 1993; Valle-Cornavaca, 2015). Una época marcada por el final de los modelos de crecimiento basados en el mercado interno y de sustitución de importaciones, y el inicio de la liberalización de algunas economías; así como una década de movimientos guerrilleros y duros enfrentamientos en Centroamanoérica y los países andinos.

En el caso específico de Perú, se había experimentado un constante crecimiento del sector entre 1950 y 1980. Un turismo que había sido beneficiado por carreteras, conectividad aérea, así como una activa publicidad estatal y privada en el mundo; además de las ventajas del propio boom de turismo norteamericano y europeo hacia el hemisferio sur, animado por la navegación aérea moderna y la intensa labor de los tour operadores (Armas, 2018). Especializado en turismo cultural, se había organizado un eje en el Surandino, rico en recursos patrimoniales en Cuzco, Puno y Arequipa. Existían otras regiones de atracción, como la propia capital, Lima y sus alrededores; y también la región norte, la sierra central y la Amazonía, aunque estas últimas embrionarias para el turismo receptivo. En 1982, luego de ingresar mayormente por el aeropuerto internacional de Lima, el grueso de este turismo se iba a Cusco (47.3\%), a Arequipa (8.9\%) o a Puno (5.6\%), como lo establece Rodríguez (1984, p. 40).

El crecimiento del sector era evidente igualmente en términos cuantitativos. Los empleos directos eran de 21 456 en 1980, los establecimientos hoteleros eran 1269, con 34618 habitaciones y 60849 camas. Un total de 239 agencias movilizaba al turismo receptivo e interno, aunque 11 manejaran el 90\% del primero, en dependencia de los operadores del exterior (Fondo de Producción Turística [FOPTUR], 1990; Rodríguez, 1984, p. 70). Se había alcanzado un cierto nivel de calidad internacional en algunos servicios en las zonas de mayor demanda, gracias a una fuerte inversión, que era atribuido a la política estatal basada en regímenes de subsidios de tipo tributario y crediticio. La crisis económica y política de 1974-1978, no había hecho mayor mella en el sector y en 1980 el país estaba en crecimiento y de retorno a la democracia -bajo el gobierno de Fernando Belaúnde 
Terry (1980-1985)-, luego de años de una dictadura militar. En diarios de circulación nacional (como Expreso, 26-10-1980, p. 27) se daba cuenta de promesas de nuevas inversiones públicas, apertura económica, así como leyes para atraer las inversiones privadas. Muchos pensaban que solo había que continuar por el sendero trazado.

Pero el mismo día en que Belaúnde ganaba las elecciones, 18 de mayo, Sendero Luminoso, organización comunista nacida en Ayacucho, empezaba su accionar armado, desatando progresivamente una violencia que sumió al país en la incertidumbre. El mundo, hacia 1981, entró en una recesión económica, que llevó a una brusca caída de los términos de intercambio a favor de los países desarrollados y en desmedro de la periferia del mundo. Sus consecuencias más notorias fueron las recesiones profundas de las economías latinoamericanas, la crisis de las deudas externas y las políticas de ajustes fiscales y monetarios que agudizaron esta crisis. Por consiguiente, el flamante nuevo gobierno democrático se estrelló contra estas realidades, que sumado a la imprevisión para tomar medidas inmediatas frente a los desafíos económicos y políticos, y al deterioro de las condiciones sociales, signó con el fracaso su administración.

Gascón (2005, p. 52) en concordancia con los aportes de O’Hare y Barret (1997) ve en la violencia subversiva como la responsable fundamental en la subida o bajada del turismo receptivo en la década de 1980. Las declaraciones del líder de Sendero Luminoso, Abimael Guzmán, en el sentido que el turismo es el símbolo del capitalismo y representa a los países ricos, y que por ello, atacarlo es atacar al gobierno, ha servido también para corroborar la importancia al relacionarlos (Wall, 1996, p. 177). Existe una noción general que asocia la caída del flujo del turismo receptivo y el terrorismo como responsable de tal crisis. Por su lado, Fuller (2008, p. 125), aunque reconoce el efecto de la recesión -sin considerar otras variables sociales o de política económicajunto al terrorismo, asegura que el flujo de visitantes extranjeros se volvió «casi inexistente» en la década de 1980. Empero, hay que precisar, estos trabajos solo hacen referencias generales, pues no hay estudios específicos al respecto.

El terrorismo no impactó en la magnitud que se pensaba en el Surandino, así como es imprescindible considerar los problemas políticos, la criminalidad, la crisis económica o lo sinuoso de las políticas públicas para entender al sector en el periodo, se planteó después de estos aportes (Armas, 2018). Lo que concordó con los datos de Loayza (2018) sobre el terrorismo de la región del Cusco. Desde entonces no se ha producido un mayor avance al respecto, aunque ha proseguido en el imaginario colectivo la percepción sobre el terrorismo y su impacto decisivo en el turismo. ${ }^{1}$

Tomando en cuenta la necesidad de ubicar en perspectiva histórica la relación objetiva existente entre el desenvolvimiento de la actividad turística y el entorno del país marcado por la violencia terrorista, las recurrentes crisis económicas y diversos factores sociales complementarios, este trabajo tiene como objetivo discernir sobre el impacto real de la violencia terrorista en el turismo receptivo en el Perú, ahondado en la preocupación del autor de este artículo desde 2018. ${ }^{2}$ El marco temporal es de 1980 a 1992, entre el inicio de la violencia terrorista

Existe un artículo del año 2020, de Miriam Menchero Sánchez, que resume en buena medida nuestro trabajo y principales conclusiones de 2018. No lo consideramos por ser reiterativo en esta evaluación.

2 La redacción de este artículo fue producto de una investigación puntual para la Universidad de San Martín de Porres entre los años 2017 y 2018 , y entregado el 28 de febrero de 2018. Se publica con ligeras actualizaciones. 
en el Perú y la captura de la cúpula de Sendero Luminoso, que marca el declive del fenómeno; periodo que también es el marco de las mayores crisis económicas del país, sucesivas, hasta que a partir de 1993 el país entra en una etapa de crecimiento económico sostenido.

Como hipótesis se plantea que la actividad turística receptiva se vio afectada por múltiples factores complementarios: el escenario económico mundial, las políticas públicas gubernamentales -particularmente las económicas- o la realidad social, donde el terrorismo fue un factor agregado, aunque permanente en el imaginario en el exterior e interior, en un contexto de peculiaridades locales.

En principio, se explica cómo las crisis económicas de aquéllos años se relacionaron con el comportamiento del sector turístico; luego, se plantea esta misma relación con la violencia terrorista desatada en la época. Ello permite valorar estos hechos y otros de índoles social en su afectación al turismo, que será materia de otro apartado, donde se aprecie además si realmente todo ello se dio de manera dramática en términos cuantitativos. Posteriormente, se establece cómo al final del periodo estudiado las cifras del turismo receptivo se deterioran de forma acelerada, describiendo sus razones reales. Por último, se presentan los resultados y las conclusiones para validar la hipótesis de trabajo.

\section{Crisis económica y turismo}

En los Estados Unidos, desde 1979, la Reserva Federal desarrolló una política monetaria restrictiva, que buscó reducir la inflación y la brecha comercial externa, estabilizando la economía. Provocó en lo inmediato una recesión de su economía para, luego de 1983, empezar un ciclo expansivo. El proceso fue seguido por una creciente liberalización, cuyos hechos fueron simultáneos también en Gran Bretaña y otros países europeos. El efecto de las medidas fue devastador sobre América Latina, pues sus países venían de una época de crecimiento basado en modelos de sustitución de importaciones -a excepción de Chile y países pequeños-, buenos precios de las materias primas exportables, y creciente endeudamiento externo. El alza de las tasas de interés llevó desde 1982 a la llamada Crisis de la Deuda Externa, iniciada en México y seguida en muchos otros países. También llevó a una tendencia decreciente de los precios de las materias primas, hasta 1987. A ello se sumaron los desequilibrios macroeconómicos internos, el contexto político, y el fracaso tanto de los programas de estabilización asistidos por el Fondo Monetario Internacional y el Banco Mundial, como de los modelos de estabilización heterodoxos implementados en Brasil, Argentina o Perú, que llevaron a esos países a violentos procesos hiperinflacionarios. El resultado fue una «década perdida» para América Latina. En términos regionales la pobreza creció, disminuyó la clase media y la informalidad económica se disparó (Bulmer-Thomas, 1998; Marichal, 2010).

En el caso del Perú, el nuevo gobierno de Fernando Belaúnde, desde fines de 1980, emprendió un cambio en el modelo de crecimiento y desarrollo, de uno que había descansado en el mercado interno y la industrialización, a otro basado en los mercados externos y la desrregulación económica. Sin embargo, los efectos fueron nocivos desde 1981, con un creciente déficit comercial, recesión industrial, a lo que se unió la crisis mundial ya expresada. Desfinanciadas las cuentas públicas, la emisión monetaria fue una salida, lo que llevó a un crecimiento inflacionario. En 1983 el programa de ajuste ortodoxo auspiciado por el FMI fue de tal magnitud que llevó al PBI a una 
brusca caída de -9\%, con el colapso generalizado del aparato productivo, a lo cual se agregó el impacto de un fenómeno de El Niño sobre la costa peruana. El ajuste de precios solo avivó la inflación y precipitó la indecisión política. El resultado fue una recesión prolongada hasta mediados de 1985 (Crabtree, 2005, pp. 49-57; Parodi, 2005, pp. 151-183).

Como puede apreciarse en la Tabla 1, los hechos mundiales y locales narrados impactaron sobre el flujo de turismo receptivo, provocando una caída sostenida entre 1980 y 1983, a lo cual debe agregarse las noticias que llegaban a diversos países acerca del comienzo de la violencia terrorista. Igualmente, habría que anotar otros hechos puntuales, como la inicial sobrevaloración del sol peruano (moneda local entre 1981 y 1982), que volvió relativamente caro el destino, con respecto a otros similares para algunos viajeros sudamericanos. Ello se refleja en la caída de los visitantes de países vecinos (de 116946 a 94040 entre 1980 y 1981) y de los Estados Unidos, aunque al principio se sostuvo el flujo proveniente de Europa (Rodríguez, 1984, p. 40). En 1983 las cifras mostraron una caída mayor, en medio de la profunda recesión de ese año y un aumento del terrorismo, que debieron disuadir a los visitantes.

Tabla 1

Perú. Turismo receptivo, 1980-1993

\begin{tabular}{ccc}
\hline Año & Ingresos & Ingreso de divisas* \\
\hline 1980 & 372790 & 292 \\
1981 & 334819 & 262 \\
1982 & 316873 & 251 \\
1983 & 273324 & 210 \\
1984 & 278783 & 215 \\
1985 & 299958 & 300 \\
1986 & 303681 & 357 \\
1987 & 330110 & 341 \\
1988 & 359281 & 448 \\
1989 & 333594 & 402 \\
1990 & 316871 & 420 \\
1991 & 232012 & 225 \\
1992 & 216534 & 156 \\
1993 & 271901 & 331 \\
\hline
\end{tabular}

Nota. FOPTUR, 1990; MITINCI 1993; Organización Mundial del Turismo (OMT), 1995; Observatorio Turístico del Perú, 2018.

* Divisas en millones de dólares. 
A partir de 1984 el turismo receptivo dejó de caer. La recuperación de las economías de Estados Unidos y Europa; el recrudecimiento de la recesión peruana, que maxideprecia la moneda local -20\% en términos reales, atractivo para los visitantes sudamericanos-; tanto como la reacción de las autoridades peruanas que, a través de las entidades encargadas de las políticas públicas (Ministerio de Industria y el FOPTUR) invierten sumas de dinero en campañas publicitarias en el exterior, explican este resultado (MICTI, 1985). También hubo un esfuerzo concertado de los gobiernos y empresarios locales con algunas compañías aéreas internacionales. El objetivo de estas políticas era salvaguardar el turismo receptivo hacia el Surandino y buscar ampliar el turismo nacional.

De todas maneras hubo estrechez económica por parte del Estado, que impidió que se prosiga con eficacia muchas obras de infraestructuras básicas (carreteras, mejoras de aeropuertos, etc.), y turísticas, por ejemplo, las previstas por el Plan Copesco, plan para el desarrollo de la infraestructura turística del Surandino, o por EnturPerú, la empresa de hoteles del Estado (Armas, 2018).

También hubo, propio de épocas de crisis, enfrentamientos entre los principales actores del sector: Estado central, autoridades locales, y empresarios. Así, sobre Cusco -más de la mitad que los que la visitaban eran extranjeros (Observatorio Turístico del Perú, 2018)- los empresarios nacionales y regionales no solo se quejaban de los constantes reajustes, por la inflación, de las tarifas de transportes aéreos o terrestres, o de los pagos por los ingresos a los sitios turísticos como Machu Picchu; sino que también los gobiernos locales pedían mayores recursos al Estado central para ejecutar una serie de obras, en particular el alcalde provincial de ese entonces Daniel Estrada, que se distinguió por sus desencuentros con las autoridades de la capital. Poco servían los esfuerzos del Viceministerio de Turismo para lograr consensos entre todos (como figura en las noticias de El Comercio, 4-10-1983, p. 8, 4-2-1984, p. 6 y 4-2-1985, p. C2 y Expreso, 7-6-1984, p. 21).

En julio de 1985 asume el poder un nuevo gobierno, el de Alan García Pérez, representante del Apra partido político de larga trayectoria-. El país estaba en recesión y además la violencia terrorista arreciaba y para enfrentar lo primero, y en el contexto del éxito inicial de planes heterodoxos de estabilización en Israel, Argentina y Brasil, el gobierno lanza uno que pretende por un lado bajar la inflación pero, por otro lado, hacer crecer la producción y el empleo sobre la base de una protección al mercado interno y aumento del consumo. Es lo que se conoció popularmente como modelo heterodoxo, un giro macroeconómico con respecto al liberalismo anterior e implicó alejarse del sistema financiero internacional. Inicialmente hubo un crecimiento económico sostenido en 1986 y 1987 -el PBI creció 10\% y 7\% respectivamente-, los salarios crecieron, se contuvo la pobreza urbana y rural -esto último gracias a grandes subsidios y la creciente demanda urbana-, y la industria ocupó su capacidad ociosa; pero a costa de un deterioro de las cuentas de las empresas públicas -petróleo, electricidad, agua, teléfonos- y del sector fiscal, debido a un congelamiento de precios y bajada de impuestos. Hacia mediados de 1987 el modelo estaba agotado, y la impericia de los autores de política económica, más un frustrado intento de estatización de la banca en 1987, terminaron polarizando a la sociedad, retrasando además el ajuste económico, desde marzo de 1988 ortodoxo, aunque signado por el gradualismo. Nuevamente, y como con el anterior régimen, la incoherencia del camino seguido llevó a un desequilibrio macroeconómico mayor, pues la subida de precios no fue seguida necesariamente por una restricción monetaria -el Estado necesitaba ingentes recursos- llevando al país a una hiperinflación y recesión desde 1988 hasta 1990. La inflación fue de 1722\% en 1988, 2775 en 
1989 y 7647\% en 1990. La economía decreció -25\% entre 1987 y 1990 (Crabtree, 2005, pp. 57-110, 187-231; Parodi, 2005, pp. 185-241).

Este panorama impactó sobre el turismo receptivo. En los años iniciales el crecimiento del sector, visible desde 1984, se aceleró hasta 1988. El turismo proveniente de los países vecinos tanto como de los Estados Unidos y de Europa suben en sus cifras. No solo es el crecimiento económico local y regional momentáneo Brasil, Argentina o Chile crecen-, sino además otros factores importantes como la política de subsidios internos del gobierno que abarata los costos (combustibles y transportes) para los operadores así como una campaña publicitaria internacional del FOPTUR, e interés de las compañías aéreas mundiales Iberia, Lufthansa, etc., por promocionar el país. Sin embargo a partir de 1989, ya en el escenario crítico, las cosas se complican. Los constantes ajustes de precios reducen ciertas ventajas anteriores. Las noticias sobre la recesión e hiperinflación asustan, tanto como la violencia; además se implementa un impuesto de cien dólares de la época -cuatrocientos cincuenta hoy- por cada turista, cobrado en los puntos de salida -aeropuerto de Lima y puestos terrestres fronterizos-, que ante la protesta de los gremios empresariales del turismo se retira a fines de ese año (noticias de El Comercio, 23-1-1989, p. C2 y 16-12-1989, p. A6). Con escasos recursos fiscales tanto el Estado y FOPTUR no pudieron mantener la política publicitaria.

Las consecuencias de la estrechez económica y la caída de los ingresos agudizaron una vez más las disputas entre los actores del sector. El Estado central fue objeto de críticas por dos aspectos: el descuido en la conservación de lugares arqueológicos o la calidad de los servicios que ofrecían sus empresas ligadas al sector - en un contexto inflacionario estas tenían muchas dificultades en mantener la calidad de sus servicios-, y por el alza de tarifas o impuestos que afectaban a las empresas o turistas. Sobre lo primero, en Cusco, particularmente Machu Picchu, el Camino Inca, o el entorno natural fueron objetos de debates por los descuidos en su conservación, mantenimiento y preservación, que involucraron a colectivos locales y a la propia Unesco. También hubo debates sobre los servicios aeroportuarios estatales en esa ciudad, por su atención deficiente al turista o descoordinación en los vuelos e informes, a pesar que recibía un promedio de 1500 turistas al día. El empresariado nacional igualmente se quejaba de los problemas de transporte aéreo a Cusco, de las deficiencias de los servicios ferroviarios en el Surandino, de los hoteles de EnturPerú y del transporte de buses obsoletos en Machu Picchu. Las quejas se multiplicaron acerca de otros problemas, como los agentes disputándose a los turistas a la salida del aeropuerto o sitios arqueológicos, las estafas de agencias de tours, etc. (Alfaro, 1991; El Comercio, 19-1-1986, p. A15, 96-1986, p. C2, 19-10-1986, p. A2, 28-1-1988, p. B12, 1-2-1988, p. B8, 30-9-1989, p. A16; CANATUR, 1989, 1990; Maxwell, 2012).

En el segundo aspecto, los impuestos nacionales y los locales -que muchos municipios desde 1988 les cobran a los turistas, para aumentar sus ingresos corrientes- el empresariado los rechazó, de manera sistemática a lo largo de los años, particularmente el de 100 dólares revisado. Pero también los usuarios masivamente rechazaban las naturales alzas -por la inflación- de las tarifas para visitar Machu Picchu o el Camino Inca, por ejemplo, por más que las autoridades locales explicaron que solo así se podían realizar los trabajos de restauración, recuperación y mantenimiento; como también se protestaba contra las tarifas de los hospedajes, sobre todo si eran públicos; o contra el alza del boleto turístico en Cusco, que daba la opción de visitar diversos monumentos (CANATUR, 1989, pp. 17-21 y 1990, pp. 12-21; noticias de El Comercio 21-1-1987, p. A16; 1-8-1987, p. 
A14). Incluso un articulista tituló: «Quieren matar turismo en Cuzco» (noticia de El Comercio 14-11-1988, p. A12), pues tales pagos eran calificados como una exacción.

También, dentro de esa ola de enfrentamientos, hubo desencuentros a nivel interregional. Por ejemplo, en 1988 hubo uno entre cusqueños y arequipeños, por el intento estatal de trasladar un teleférico a Machu Picchu, desde la obra hidroeléctrica de Charcani V, que provocó una reacción de los arequipeños que vieron el potencial turístico en su uso y de los mismos cusqueños, donde había crecido el rechazo a la colocación de un teleférico o al asfaltado de una carretera hasta Machu Picchu, considerándose que atentarían contra su integridad natural (La noticia lo recoge el diario El Comercio 9-1-1987, p. A15 y 26-2-1988, p. A16).

Así, los efectos de una crisis económica deben medirse, no solo desde el punto de vista cuantitativo, sino también desde el punto de vista de las tensiones que produce, el deterioro de las condiciones sociales, los servicios ofrecidos y su impacto general sobre el sector.

\section{Terrorismo y turismo}

A los problemas de la crisis económica, vigentes casi a lo largo de toda la década, se sumó la violencia subversiva. Como ya se dijo, en 1980 el Partido Comunista del Perú Sendero Luminoso, de orientación maoísta y nacido en Ayacucho -en la región centro-sur-, empieza su accionar violento. Este tenía como objetivo la destrucción del orden democrático y de las instituciones políticas del país. Aunque al inicio el número de atentados fue pequeño, conforme avanzaron los años estos fueron aumentando tanto como las víctimas y hacia 1982 se convirtió en un problema nacional prioritario. En 1981 el presidente Belaúnde declara el estado de emergencia en varias provincias de Ayacucho, pero ante el aumento de atentados y asesinatos de políticos o dirigentes sociales, en diciembre de 1982 se entregan diversas provincias de los departamentos de Ayacucho, Huancavelica y Apurímac al control político y militar de las fuerzas armadas. Ya para entonces la acción terrorista de atentados y asesinatos se había expandido a la sierra y selva central, y a ciertos lugares de la sierra norte e incluso a la capital y al propio Surandino: Arequipa, Cuzco y Puno. Sin embargo, hay que advertir que diversos colectivos regionales sociales - desde organizaciones de izquierda hasta la Iglesia católica- posibilitaron que Sendero Luminoso no avanzara tanto como se hubiera esperado en Puno y Cusco (Comisión de la Verdad y Reconciliación [CVR], 2004, pp. 77-96 y 207-242; Degregori, 2011).

Hacia 1984 se une a la acción armada el Movimiento Revolucionario Túpac Amaru (MRTA), marxista-leninista, complejizando el panorama. El Estado se mostró rebasado y su acción, a veces no bien estructurada, llevó a situaciones de violación de los derechos humanos. Con el nuevo gobierno de Alan García la violencia siguió recrudeciendo, esparcida por los lugares señalados. En 1986 el gobierno implementa el toque de queda en la ciudad de Lima, que duró más de un año, buscando disminuir los constantes atentados -que provocaban cortes de energía eléctrica y de agua- y asesinatos. La llamada matanza de los penales (en junio de 1986) fue un hecho calamitoso que saltó, como otras masacres o descubrimientos de fosas comunes, a la escena internacional. Entre 1988 y 1990 la violencia se desbordó y los «paros armados» tanto como las otras formas subversivas de ataques se vuelven cada vez más recurrentes, en un contexto -no lo olvidemos- de hiperinflación e hiperrecesión. En total, la CVR ha establecido en más de 69000 los fallecidos por la violencia, la mayor parte en la década aludida. 
En la práctica, el poco turismo receptivo existente en Ayacucho, como en la sierra central así como en la selva central, desaparece en medio del conflicto armado, los permisos previos, las requisas, toques de queda y otros elementos que acompañaron durante años la vida de los pobladores de aquellos lugares. En este proceso en ascenso, al inicio las entidades vinculadas al turismo -prensa, gremios y entidades estatales- buscaron minimizar su impacto. La focalización del fenómeno en Ayacucho y alrededores parecía darles la razón (Figura 1). Pero que no avanzara hacia el Surandino -la región receptora de turismo por excelencia- en la magnitud como lo hizo en otras regiones no quiere decir que no haya tenido cierta presencia, como se muestra en la Tabla 2, y sobre todo, que no haya creado una atmósfera de incertidumbre y permanente situación de alerta entre los actores regionales y el Estado.

\section{Figura 1}

Perú 1980-1992. Porcentaje de muertos y desaparecidos reportados a la CVR por regiones

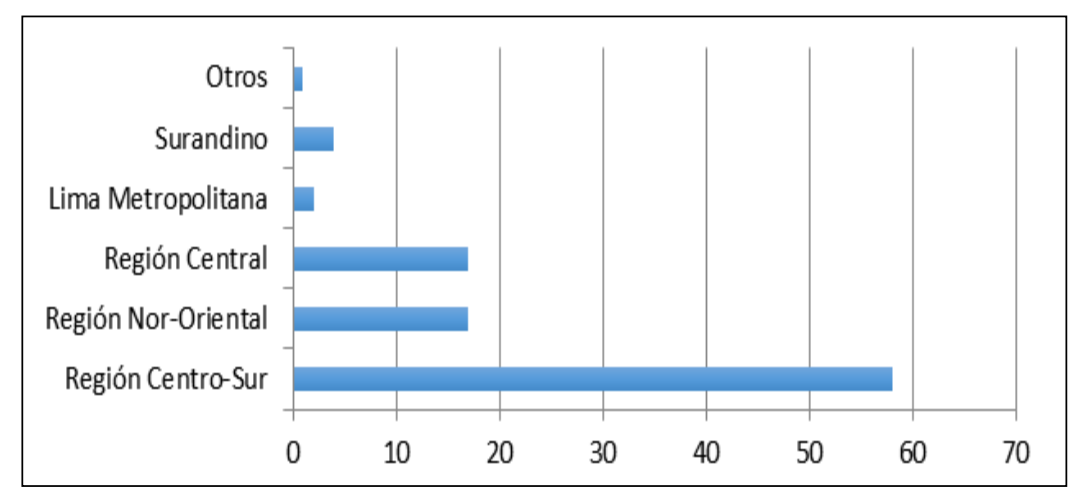

Nota. Adaptado de CVR, 2004, pp. 80 y 97.

Tabla 2

Número de atentados en Cusco y departamentos aledaños, 1980-1989

\begin{tabular}{lccccccccccc}
\hline Años & \multirow{2}{*}{1980} & 1981 & 1982 & 1983 & 1984 & 1985 & 1986 & 1987 & 1988 & 1989 \\
\hline Apurímac & 9 & 17 & 53 & 12 & 23 & 17 & 139 & 107 & 140 & 68 \\
Cusco & 8 & 75 & 29 & 32 & 30 & 68 & 69 & 47 & 35 & 30 \\
Madre de Dios & & & 1 & & 1 & & 1 & 2 & 1 & \\
\hline
\end{tabular}

Nota. Adaptado de Alfaro, 1991, p. 206. 
La zozobra fue lo que directamente afectó al turismo receptivo, es decir, la posibilidad de un atentado donde cualquier viajero podía terminar siendo la víctima. Abonaron a ello algunos ataques específicos que involucraron al turismo y que se dieron a lo largo de la década. En mayo de 1984, en la ciudad de Trujillo, hubo un atentado contra el hotel de Turistas -perteneciente a la cadena hotelera estatal EnturPerú-. En la víspera de la Navidad de 1986 y en pleno estado de emergencia en Lima, se produjo una explosión en el estacionamiento del aeropuerto internacional Jorge Chávez, dejando vehículos en mal estado. En 1986 se produjo una incursión senderista a un hotel en Trujillo, y en agosto de 1987 estalló un coche bomba en el estacionamiento del Hotel Sheraton de Lima, produciendo una situación de alerta extrema y angustia para los viajeros al no sentirse seguros en su lugar de hospedaje; y tampoco en los lugares de destinos. Igualmente, fue muy comentado por la prensa un suceso ocurrido en diciembre de 1988, cuando un grupo de senderistas asaltó y quemó el puesto de control del Parque Nacional del Huascarán (El Comercio, 8-5-1984, p. 11, 24-12-1985, p. 1, 26-8-1987, p. A14, 9-7-1987, p. A12 y 1-12-1988, p. A13 y Expreso, 23-3-1986, p. 29). A veces, incluso, era una acción más directa y brutal, como el atentado en la estación de tren de San Pedro en Cusco, en 1986, donde murieron varios turistas; o los que sufrieron los alcaldes de Cusco y Nueva Jersey, en diciembre de 1988, en la ruta a Machu Picchu. También, impresionaron a la prensa y a los operadores a nivel internacional los asesinatos de cooperantes internacionales en las comunidades aledañas a Cusco (Alfaro, 1991, pp. 202, 204; Loayza, 2018).

Aunque los atentados los involucraban puntualmente, lo más importante fue la respuesta de miedo de turistas, probablemente lo que buscaban los subversivos. En Arequipa los atentados y el miedo explicaban en buena medida -según un funcionario de turismo- el descenso del turismo en 1983. A ello se sumaba la delincuencia común que, en el contexto de inestabilidad económica y política, se agravó en la ciudad de Lima y en otras ciudades: robos en las calles o en los aeropuertos, asaltos impunes incluso en sus hospedajes o en restaurantes. En declaraciones a los medios, Carlos Heckmann, gerente general de la línea aérea Varig, señalaba que el problema se debía a una sensación de falta de seguridad, aunada a la postración económica y a la criminalidad común, que hacía que muchos turistas no se atrevieran a desplazarse con tranquilidad (El Comercio, 17-9-1983, p. C2 y 1-1-1984, p. 16). Miedos colectivos exacerbados por hechos concretos, atizados por la amplificación de las noticias a nivel mundial y la poca capacidad de distinguir las complejidades y las realidades peruanas, lo cierto es que la imagen del Perú en el mundo se convirtió en deplorable y desalentaba la llegada de algunos - estimulados por las recomendaciones que ciertos gobiernos hacían a sus ciudadanos-; aunque también debemos relativizar esto. Se leía en el principal diario del país a fines de 1989: «el terrorismo es un fenómeno a nivel mundial, su efecto en la imagen del país es relativa... No podemos decir que el terrorismo ha influido en retraer el turismo receptivo, pero tampoco se puede decir que no afecta» (Terrorismo, la imagen y el turismo, 1989, p. C1).

\section{El no derrumbe del turismo receptivo}

En un contexto de crisis económica, aumento exponencial del subempleo, informalidad y poca capacidad del Estado para hacer cumplir las leyes y garantizar el orden, la criminalidad común -robos, asaltos, secuestros- se multiplica en el país, aunada a la propia labor de la prensa de remarcarla y cuestionar con ello la capacidad de los organismos gubernamentales para cumplir con su labor. Los turistas extranjeros -aunque también nacionaleseran presas fáciles, pues en el imaginario local el visitante es sinónimo de portador de artefactos caros que fácilmente se pueden revender en el mercado negro; uniéndose a las sensaciones de inseguridad que provocaban 
en los viajeros otros fenómenos masivos, como la informalidad y la pobreza, con las que se topaban (noticias de El Comercio, 4-2-1987, p. A4, 4-1-1988, p. A13, 1-12-1988, p. A13, 18-1-1989, p. A9, 6-11-1989, p. C2).

Sin embargo, el terrorismo, la crisis económica o la pauperizacion social no lograron inicialmente derrumbar dramáticamente al turismo receptivo. Si bien en toda la década los números de llegadas no crecieron, tampoco se dio el caso de lo ocurrido en otros países del mundo, donde por guerras internas o externas prácticamente desapareció; o en Colombia, donde por problemas económicos, violencia y otros asuntos conexos entre 1979 y 1983 el turismo receptivo se derrumbó en -50\%; o en Brasil, donde por la crisis económica cayó en -40\% aproximadamente entre 1986 y 1990 (MITINCI, 1993). Si se aprecia la Tabla 1, en el caso peruano entre 1980 y 1983 el turismo receptivo solo cayó en un cuarto del total, para recuperarse en un 28\% entre 1984 y 1988, y descender apenas un 7\% en 1989. A pesar del terrorismo, informalidad, violencia cotidiana, dificultades aéreas o de carreteras, el turismo receptivo no se vino abajo en forma estrepitosa. En la respuesta está el parcial impacto del terrorismo en el Surandino, tanto como las propias dinámicas mundiales de los flujos, la situación de los países de origen, la ventaja que ofrecía el Perú -a pesar de las quejas en sentido contrario- de costos bajos comparados con otras realidades -reforzados por los fuertes subsidios a los servicios públicos, combustibles e insumos diversos en varios momentos de esos años-, tipo de cambio muy favorable para el dólar o monedas europeas, entre otros factores, que hicieron asumibles los costos sociales y el riesgo inherente. Desde el punto de vista de oportunidades perdidas, era por supuesto lamentable la realidad para muchos actores del turismo vinculados al flujo receptivo: el turismo de montaña prácticamente desapareció, no fue posible desarrollar circuitos y lugares alternativos al eje Lima-Surandino, se perdió el poco turismo foráneo hacia Cajamarca o la sierra central, no hubo grandes inversiones en infraestructura para atender a este tipo de turistas y las utilidades en dólares bajaron sensiblemente.

Algunos segmentos de turismo disminuyeron pero aumentaron otros. Por ello, David Griffith, presidente del FOPTUR, señalaba que los altibajos en la década se debían más a la incapacidad de una política de marketing continua y en los últimos años a la obsolescencia de la infraestructura y los problemas con la imagen del Perú, por «terrorismo, crimen y robos» (citado en Canatur, 1990, pp. 94-96). Aunque siendo parcial su respuesta da indicativos de la misma complejidad del fenómeno.

Para mayor análisis veamos lo ocurrido en la región del Cusco. Sin duda la tensión nacional, con una realidad política muy turbulenta, la desconfianza del empresariado nacional hacia el gobierno de Alan García, tanto como disputas locales y la crisis económica nacional, anunciaban una crisis del sector. Sin embargo, como se puede apreciar en la Tabla 3, los arribos de extranjeros -en consonancia con la tendencia nacional- decayeron entre 1981 y 1983, para elevarse de nuevo de 1984 hasta 1988, y luego volver a descender en 1989. No obstante, fue recién a partir de este último año que la situación a este respecto se volvió angustiante y continuó en los primeros años de la década de 1990. En cambio el turismo nacional creció en forma sostenida y sin pausa hasta 1987, libre de mayores inconvenientes. Solo la crisis económica que se inició en 1988 la afectó, aunque no bajó de un nivel de 240000 arribos hasta después de 1991. Es decir, siempre en niveles superiores a los de la década de 1970 e inicios de aquella. Campañas publicitarias, subvenciones y subsidios, entre otros elementos reseñados, explican este comportamiento interno. 
Tabla 3

Arribos a hospedajes de la ciudad del Cusco, 1980-1992

\begin{tabular}{cccc}
\hline Año & $\begin{array}{c}\text { Arribos } \\
\text { extranjeros }\end{array}$ & $\begin{array}{c}\text { Arribos } \\
\text { nacionales }\end{array}$ & $\begin{array}{c}\text { Ocupabilidad de } \\
\text { habitaciones }\end{array}$ \\
\hline 1980 & 158732 & 159870 & 47.10 \\
1981 & 142314 & 177361 & 44.98 \\
1982 & 131791 & 193634 & 29.68 \\
1983 & 112234 & 195753 & 31.50 \\
1984 & 126827 & 239058 & 35.39 \\
1985 & 116955 & 250011 & 32.74 \\
1986 & 144853 & 282912 & 42.30 \\
1987 & 153372 & 329953 & 41.02 \\
1988 & 168052 & 277640 & 39.19 \\
1989 & 106896 & 240204 & 28.58 \\
1990 & 68512 & 242804 & 24.65 \\
1991 & 109848 & 310408 & 25.81 \\
1992 & 45734 & 196530 & 21.57 \\
\hline
\end{tabular}

Nota. Adaptación de MITINCI, 1993, tomo II.

Otra manera de comprobar el dinamismo cusqueño, por lo menos hasta 1988, es percatarse de la inversión que se registró en la infraestructura turística. Lo que también permite comprender parcialmente que la ocupabilidad fue descendiendo en la década.

\section{Tabla 4}

Hospedaje en Cusco, 1985-1991

\begin{tabular}{lccccccc}
\hline Año & 1985 & 1986 & 1987 & 1988 & 1989 & 1990 & 1991 \\
Establecimientos & 105 & 107 & 141 & 153 & 164 & 171 & 170 \\
\hline
\end{tabular}

Nota. MITINCl, 1993. 
De acuerdo con Alfaro, utilizando otras cifras y clasificaciones, el número de hospedajes en Cusco pasó de 47 en 1985 a 191 en 1992 (Alfaro, 1991, p. 161). Según una cifra alterna, existían 162 hospedajes al final de la década, 6.92\% de toda la oferta nacional (2007 establecimientos) con 3486 habitaciones y 6713 camas; veintinueve de primera categoría. La estacionalidad de esta infraestructura era alta en diciembre-enero, en julioagosto y en octubre.

Así pues, alejada parcialmente de los impactos directos de la violencia terrorista, la región vivió una época de relativa tranquilidad hasta 1988, no exenta de disputas y problemas locales diversos. ${ }^{3}$ Recién hacia 1989 empezó a sentirse la crisis del sector, agobiado por las restricciones del turismo receptivo en sus países de origen para viajar al Perú -por la imagen de inseguridad y también de otros problemas mundiales, como lo ocurrido tras el atentado en Lockerbie (Reino Unido, 1988)-, la escasez de divisas y la propia crisis económica nacional.

En las otras regiones el impacto del terrorismo sí fue determinante en el derrumbe de las cifras. En la región central, Junín (Tabla 5), se puede apreciar que la acción subversiva hizo inviable el desarrollo del turismo receptivo en dicho lugar. Solo el turismo nacional permitió mantener la actividad local, salvo en 1989, año de fuerte hiperinflación e hiperrecesión.

Ayacucho tuvo que soportar una ausencia casi total de visitantes. El estado de emergencia, las muertes y desapariciones forzadas eran poderosas razones para disuadir a cualquiera de visitar el lugar. Aunque igual algunos pudieron atreverse. Galindo Santos, expresidente de la comisión departamental de turismo, sostenía (en El Comercio 16-2-1983, p. 16) que los principales factores de la disminución eran la situación de emergencia de las provincias de Ayacucho y el explicable temor frente a los fenómenos naturales -ese año el fenómeno El Niño se presentó-.

Cajamarca, que sufrió también los estragos de la subversión, aunque no a los niveles de Ayacucho, vio igualmente la casi desaparición de los pocos turistas extranjeros que antes la visitaban -era, sin embargo, como Ayacucho, todavía un destino embrionario-.

\section{El derrumbe del turismo receptivo entre 1990 y 1992}

Casi a poco de la llegada al poder, el gobierno de Alberto Fujimori (1990-2000) realizó, de la mano del nuevo equipo económico y bajo el consejo de los organismos financieros internacionales (Banco Mundial, Fondo Monetario Internacional), un programa de ajuste económico conocido como Fujishock, que se dio el 8 de agosto de 1990, y luego un duro programa de estabilización, que se prolongó hasta fines de $1992^{4}$. Política monetaria contractiva, altas tasas de interés y casi nula inversión pública caracterizaron esos iniciales años noventa.

\footnotetext{
Lo cual no quiere decir que no hubiera zozobra. Mayormente los atentados eran en zonas altas de la región, alejadas de los lugares de interés (Loayza, 2018). El mencionado autor aisla esta causa para entender la caída del turismo regional entre 1980-1983, previo al crecimiento de 1984-1988.

4 A fines de la década de 1980 e inicios de la siguiente se produjeron en diversos países latinoamericanos duros ajustes económicos, seguidos de programas de estabilización ortodoxos y aperturas económicas. Siguiendo los lineamientos del llamado Consenso de Washington (1989), los países de la región se anclaron en modelos de crecimiento basados en los mercados externos y en la reprimarización de sus aparatos productivos (Moreno-Bird et al., pp. 345-365; Williamson, J., 2004, pp. 1-14).
} 
Tabla 5

Arribos a Junín, Ayacucho y Cajamarca, 1980-1992

\begin{tabular}{ccccc}
\hline Año & $\begin{array}{c}\text { Arribos } \\
\text { extranjeros } \\
\text { a Junín }\end{array}$ & $\begin{array}{c}\text { Arribos } \\
\text { nacionales } \\
\text { a Junín }\end{array}$ & $\begin{array}{c}\text { Arribos } \\
\text { extranjeros } \\
\text { a Ayacucho }\end{array}$ & $\begin{array}{c}\text { Arribos } \\
\text { extranjeros a } \\
\text { Cajamarca }\end{array}$ \\
\hline 1980 & 21892 & 512911 & 198 & 1467 \\
1981 & 13244 & 390051 & 91 & 1230 \\
1982 & 12792 & 388539 & 17 & 1331 \\
1983 & 7245 & 371327 & 9 & 439 \\
1984 & 7369 & 381676 & 3 & 823 \\
1985 & 6756 & 413691 & 5 & 189 \\
1986 & 4759 & 487290 & 0 & 215 \\
1987 & 2762 & 499999 & 0 & 110 \\
1988 & 3053 & 552641 & 7 & 490 \\
1989 & 1640 & 175112 & 108 & 8 \\
1990 & 1352 & 494812 & 40 & 60 \\
1991 & 1052 & 372280 & 152 & 218 \\
1992 & 258 & 410254 & 32 & 0 \\
\hline
\end{tabular}

Nota. MITINCI, 1993, tomo II.

La inflación fue descendiendo, pero al costo de prolongar la recesión económica. Desde el punto de vista de la economía familiar, a la caída del salario, visible desde 1988, le siguió el alza en términos reales de los precios domésticos y por lo tanto una disminución de la capacidad de consumo. La liberalización de la economía apertura comercial, cambiaria, financiera, etc.-, complementó el panorama, cuyas consecuencias más evidentes en el corto plazo fueron el derrumbe de las industrias del mercado interno y un mayor aumento del subempleo y de la informalidad (Arce, 2010, pp. 31-87; Parodi, 2005, pp. 243-300; Wise, 2003, pp. 233-288).

A lo que se sumó una mayor inestabilidad social y política. Ello se produjo en tres frentes:

Por un lado, el recrudecimiento de la violencia subversiva, cuyo grupo principal, Sendero Luminoso, desarrolló hasta 1992 «paros armados» cada vez más frecuentes en la propia ciudad capital, con cortes de energía eléctrica y de agua, coches bombas, asesinatos y zozobra general -el más importante se produjo en julio de 1992 (Tarata y otros atentados)-. Esta situación, que la prensa internacional se encargaba de informar, se prolongó hasta fines de 1992, solo cediendo con la captura de los líderes de Sendero Luminoso y la ofensiva posterior del Estado.

Estas acciones cuando involucraban a turistas extranjeros eran resaltadas por la prensa. Por ejemplo, en enero de 1990, en Abancay, dos turistas franceses fueron asesinados a tiros por presuntos terroristas, cuando viajaban en ómnibus desde Ayacucho rumbo a Andahuaylas. Al año siguiente, subversivos mataron al ciudadano alemán Kuedorf Joachin Alfrecht y a otro turista colombiano, mientras viajaban de Puno a Cusco. Pero los hechos donde directamente estaban involucrados los turistas extranjeros eran escasos, solían ocurrir en zonas 
de conflicto o a veces se mezclaban con hechos imputados al crimen organizado (como se encuentran en las noticias de El Comercio, Lima, 14-1-1990, p. A1, 13-2-1992, p. A11, 21-8-1992, p. A9). En todo caso, si se sigue a esa prensa, denotó en su momento el interés por resaltar la presencia de turistas extranjeros entre los afectados, cuando en la práctica los nacionales también los fueron. Es interesante apreciar asimismo, cómo las noticias sobre turismo y violencia subversiva van desapareciendo luego de 1992, cuando tal violencia fue cediendo progresivamente, para ser reemplazadas por las del crimen organizado o la delincuencia común. No es que estas no existieran antes, sino que ganaron notoriedad (Armas, 2018).

En el verano de 1991, se desata la epidemia del cólera en el Perú, agravada por las malas condiciones de higiene de las ciudades, la pobreza e informalidad existentes. Muchas personas perecieron en esa epidemia y la noticia se esparció por el mundo; autoridades de diversos países alertaron a sus ciudadanos del riesgo sanitario inherente, lo que inhibió a muchos de visitar el país. En un informe de mayo de 1991, se decía que la pregunta era saber cuál de todas las cuestiones le hacía más daño al turismo: «se podría decir que con algunas pocas excepciones el terrorismo no ataca turistas, a diferencia de los terroristas en el extranjero». Igualmente en el caso del cólera «se han presentado 100 mil casos pero de estos solo ha muerto el 1\% y las víctimas son de barrios marginales donde no van los turistas». El problema tenía que ver con la imagen, con los temores que desataban estos sucesos. Se acusaba a los medios de prensa de responsables, pues tales noticias habían «costado mucho dinero al Perú, sus exportaciones y el turismo han sido afectados severamente» (informe aparecido en El Comercio, 27-5-1991, p. C4) . De forma más concreta, el alcalde de la localidad de Nasca, en 1991, decía que por efectos de la epidemia del cólera había bajado notablemente el turismo hacia ese lugar, y también, por las noticias de los constantes ataques subversivos en la zona (Valle, 1991, p. A3).

Por último, el tercer factor de inestabilidad del periodo fue el creciente carácter autoritario del régimen. Los constantes enfrentamientos entre el Poder Legislativo y el Ejecutivo, y otros hechos políticos, se hicieron visibles en abril de 1992, cuando se produjo el autogolpe o cierre del Congreso -así como una reorganización del Poder Judicial- por parte del gobierno. Ello llevó a una reacción inmediata de la comunidad internacional: congelamiento de préstamos y ayuda económica al Perú, reuniones de emergencia de la Organización de Estados Americanos (OEA) y finalmente una presión que forzó al gobierno a convocar a elecciones para un nuevo Parlamento (Burt, 2011, pp. 61-249; Murakami, 2007, pp. 243-341; Parodi, 2005, pp. 281-288). Todo esto impactó en el exterior y en la imagen del país.

Así pues, por una serie de acontecimientos de diversa índole, producida a lo largo de 1991 y 1992, en el turismo receptivo se aceleró la caída en sus números, cuestión que la crisis económica y la violencia de la década anterior no habían conseguido en esa magnitud, según lo que se conoce. Es más, hacia 1988 este turismo había llegado a un pico máximo. Como se aprecia en el Tabla 1, la caída iniciada en 1989 simplemente se profundizó en 1991 y 1992, trayendo como consecuencia el derrumbe de las diversas ramas de la actividad: restaurantes, transportistas, comercio artesanal, etc. En la Tabla 7 se evidencia en cifras lo que significó para los diversos establecimientos cuyo público esencial y central eran los turistas extranjeros: básicamente hoteles de tres a cinco estrellas en Lima y en diversas ciudades del Surandino. En 1992 los arribos extranjeros significaron un poco más del 27\% de los que ocurrieron en el año 1988. En la Tabla 3 se muestra su impacto en la región más importante para el turismo receptivo: Cusco. El dato es elocuente. 
Por donde se mire, los años 1990-1992 fueron fatales para el turismo receptivo. Dos indicadores adicionales permiten centrarse en 1992 como el año piso para el turismo. El turismo nacional, tan resistente a los problemas domésticos finalmente cae fuerte en ese año. Sin lugar a dudas tanto la recesión, los bajos salarios y el fin de los subsidios como el recrudecimiento de la violencia urbana inhiben a lo que antes era un riesgo asumible. Un segundo dato se evidencia en la Tabla 6, de llegadas internacionales en la época, donde se aprecia que la caída máxima se da a lo largo de 1992 y hasta enero de 1993. Mala imagen del Perú en los Estados Unidos, Europa y Sudamérica -principales regiones del mundo de las cuales proceden los turistas-, advertencia de varios países a sus viajeros sobre el Perú -como el Travel Warning norteamericano-, riesgo sanitario, encarecimiento de las transacciones financieras locales, problemas en el funcionamiento de las redes locales de la actividad son algunas de las problemáticas de fondo. Puede apreciarse que la situación se torna aguda en el segundo semestre de 1992, tras el autogolpe de abril de ese año y la agudización de la política económica recesiva. En un estudio posterior de PromPerú -la nueva agencia de promoción del turismo y la imagen del Perú- se concluyó que el turismo receptivo cayó hasta 1992, particularmente el norteamericano. En consecuencia, es posible postular que, como mínimo, se agudiza la situación por la recesión económica, la incertidumbre política generada, el terrorismo, el riesgo sanitario, y los problemas financieros derivados de todos ellos (Sector turismo y su privatización en el Perú (1994, p. 31).

Tabla 6

Llegadas internacionales al Perú, 1991-1993

\begin{tabular}{llll}
\hline \multicolumn{1}{c}{ Año } & 1991 & 1992 & 1993 \\
\hline Enero & 29915 & 23795 & 21174 \\
Febrero & 19644 & 20036 & 21066 \\
Marzo & 16000 & 18255 & 19830 \\
I trimestre & 65559 & 62086 & 62070 \\
Abril & 15162 & 15190 & 18064 \\
Mayo & 14474 & 14323 & 21228 \\
Junio & 17022 & 16571 & 21148 \\
II trimestre & 46658 & 46084 & 60440 \\
Julio & 22606 & 20718 & 26594 \\
Agosto & 21167 & 19276 & 26992 \\
Septiembre & 17494 & 14519 & 19296 \\
III trimestre & 61267 & 54513 & 72882 \\
Octubre & 16968 & 16441 & 23407 \\
Noviembre & 18808 & 16219 & 24609 \\
Diciembre & 22752 & 21191 & 31752 \\
IV trimestre & 58528 & 53851 & 79768 \\
Total anual & 232012 & 216534 & 275160 \\
\hline Nota Adapto & & 219 \\
\hline
\end{tabular}

Nota. Adaptado de Sector turismo y su privatización en el Perú, 1994, p. 31. 
Tabla 7

Índices de ocupabilidad de establecimientos de hospedajes, 1990-1995

\begin{tabular}{lcccccc}
\hline Lugar Año & 1990 & 1991 & 1992 & 1993 & 1994 & $1995(1)$ \\
\hline Lima & 45.4 & 43.6 & 41.2 & 43.9 & 45.3 & 46.6 \\
Cuzco & 24.7 & 25.8 & 21.6 & 23.8 & 29.4 & 28.5 \\
Lambayeque & 45.5 & 49.8 & 23.8 & 22.7 & 24.4 & 24.4 \\
Arequipa & 39.8 & 39.1 & 31.0 & 31.5 & 33.3 & 33.3 \\
Puno & 26.3 & 15.4 & 18.8 & 20.3 & 22.4 & 22.5 \\
Loreto & 27 & 23.5 & 26.2 & 32.1 & 36.0 & 34.3 \\
Ayacucho & 18.9 & 20.6 & 20.5 & 26.2 & 30.3 & 24.3
\end{tabular}

Nota. APOYO, 1995, p. 12.

(1) Primer semestre.

Tabla 8

Perú. Capacidad hotelera, 1980-1989

\begin{tabular}{ccc}
\hline Año & Establecimientos & Camas \\
\hline 1980 & 1269 & 60889 \\
1981 & 1384 & 65484 \\
1982 & 1569 & 70822 \\
1983 & 1523 & 71785 \\
1984 & 1627 & 74504 \\
1985 & 1666 & 76494 \\
1986 & 1702 & 78221 \\
1987 & 1749 & 79973 \\
1988 & 1913 & 86487 \\
1989 & 2032 & 89454 \\
\hline
\end{tabular}

Nota. Adaptado de FOPTUR, 1990.

\section{Conclusiones}

De acuerdo a las diversas evidencias presentadas, el turismo receptivo en el Perú sufre una caída moderada de 1981 a 1983, para luego crecer también en forma moderada hasta 1988, y caer en forma acelerada de 1989 a 1992. Para explicar estos comportamientos es necesario considerar la importancia de las políticas y desenvolvimientos de la economía mundial y local, así como en el impacto del fenómeno del terrorismo. Sobre lo primero, hay que tomar en cuenta la evolución de los ciclos económicos mundiales y locales, y la importancia de los subsidios, los impuestos, los tipos de cambios, o las capacidades de consumos. Sobre lo segundo, remarcar que el terrorismo creó inseguridades, miedos y una imagen de descrédito mundial. Como elementos complementarios, se encuentra el efecto de las políticas turísticas gubernamentales, la incidencia de la criminalidad común, la precariedad social, los problemas sanitarios y la incertidumbre política. 
Es decir, en función de los resultados no se puede señalar al terrorismo como único y directo responsable del comportamiento del turismo receptivo, que es lo que generalmente se hace. El terrorismo no necesariamente produjo derrumbes dramáticos de las cifras ni afectó decisivamente el comportamiento del área geográfica con mayor desarrollo turístico, hay otros elementos que lo explican, que crearon además peculiaridades y singularidades en la manera en que las cifras variaron; como se afirmó, hubo ondulaciones de acuerdo a las causas ya señaladas. Monti (2011, pp. 93-106), de acuerdo a otra realidad, al señalar la importancia de las estrategias gubernamentales de marketing, con atención mercados y segmentos menos afectados; oferta de productos nuevos, actualizados o más baratos; o cooperación entre los sectores públicos y privados, nos proporciona también otras razones para entender lo ocurrido entre 1984 y 1988.

Además, cuando las cifras finalmente cayeron desde 1989, fue a costa del turismo procedente de los Estados Unidos y de Europa, no de los países vecinos de Sudamérica -este sube en términos relativos de 26\% a 38.15\% entre 1989 y 1992, con un ligero retroceso en números absolutos- (Alfaro, 1991, p. 17; MITINCI, 1993). Oportunidades por precios deprimidos y riesgo asumible pueden estar entre las explicaciones. Además, y a pesar de la violencia subversiva, de la crisis económica y de la creciente informalidad e inseguridades, la infraestructura crece mucho en ese periodo. Se puede verificar al observar la Tabla 4 y la Tabla 8, donde se muestra el dinamismo de la oferta hotelera nacional en la década. En 1989, de 2032 establecimientos, 313 de ellos con 13729 habitaciones y 25498 camas se consideraban de nivel internacional. Atendían la demanda y muchas veces se formaban cuellos de botella en ciertos momentos del año. Para ese año la ocupabilidad nacional fue de 37.32\%. En cinco estrellas 20.6\%; en cuatro 8.8\%; y en tres 36.7\%, aumentando los porcentajes en los de menos estrellas (Alfaro, 1991, p. 94; CANATUR, 1984, p. 81 y 1990, pp. 69). La fuerza que entonces todavía tenía el turismo nacional, como la inversión existente -en un contexto de búsqueda de ingresos familiares complementarios- permite explicar parcialmente el fenómeno.

El turismo como ingreso complementario, así como el incentivo de las leyes promocionales, inducen a mirar con otros ojos el comportamiento de la inversión en el periodo. Si bien las grandes inversiones privadas están ausentes, para 1989 había 4314 restaurantes dedicados al turismo, y las agencias de viajes crecieron bastante, pasando de 239 (1980), a 533 (1981), 715 (1985), 1283 (1989) y 1917 (1992). El crecimiento fue espectacular en casi todas las regiones. Sin embargo, de 306 encuestadas a fines de la década, solo $22.5 \%$ se dedicaba a turismo receptivo, $13.4 \%$ a turismo interno y el resto vendía pasajes o paquetes turísticos al exterior. Es pertinente recordar que el turismo receptivo estaba concentrado entre pocos, con fuertes relaciones con los grandes tour operadores mundiales: en 1985 solo cinco de todas las agencias eran mayoristas. Para 1989 ellas representaban el 1.81\% del número total (Alfaro, 1991, p. 97; CANATUR, 1984, p. 284 y 1989, p. 10).

De esta manera, en países como Perú, el desempeño del turismo en contextos de terrorismo, crisis económica y diversas otras variables, se debe mirar con sus múltiples y complementarios elementos de intervención, evitando aislarlos, pues la complejidad histórica, cargada de tantos hechos entrelazados, impide cualquier estudio parcial.

\section{Conflicto de intereses}

El autor $\square$ o tienen conflicto de intereses con el contenido de este trabajo. 


\section{Referencias}

Alfaro, A. (1991). El turismo en la zona arqueológica del Cuzco y su impacto económico en la región inca [Tesis de grado en economía, Universidad de Lima]. Lima.

APOYO. (1995). Situación y perspectivas del sector turismo. Apoyo.

Arce, M. (2010). El fujimorismo y la reforma del mercado en la sociedad peruana. Instituto de Estudios Peruanos.

Armas, F. (2018). Una historia del turismo en el Perú. El Estado, los visitantes y los empresarios (1800-2000). Fondo Editorial Universidad de San Martín de Porres.

Aurioles, J. (2011). Crisis económica y cambios en el turismo. Mediterráneo económico, 20, 99-113.

Baumert, T. (2016). Terrorismo y turismo: una revisión de la literatura acerca de la repercusión de los atentados sobre el sector turístico. Información Comercial Española, 893, 51-70.

Bringas, N. L. y Verduzco, B. (2008). La construcción de la frontera norte como destino turístico en un contexto de alerta de seguridad. Región y Sociedad, 20(42), 3-36.

Bulmer-Thomas, V. (1998). La historia económica de América Latina desde la Independencia. Fondo de Cultura Económica.

Burt, J. M. (2001). Violencia y autoritarismo en el Perú: bajo la sombra de Sendero y la dictadura de Fujimori. Instituto de Estudios Peruanos, Asociación de Servicios Educativos Rurales e Instituto Peruano de Antropología Forense.

Causevic, S. y Lynch, P. A. (2013). Political (in)stability and its influence on tourism development. Tourism Management, 34, 145157.

Cámara Nacional de Turismo (CANATUR). (1984). Anales del IV Congreso Nacional de Turismo. CANATUR.

Cámara Nacional de Turismo (CANATUR). (1989). Situación del turismo en el Perú. https://www.canaturperu.org/

Cámara Nacional de Turismo (CANATUR). (1990). Turismo: Horizonte 2000. Anales del VI Congreso Nacional de Turismo 1990 [Acta]. https://www.canaturperu.org/

Cólera, terrorismo y turismo. (1991, 27 de junio). El Comercio.

Comisión de la Verdad y Reconciliación (CVR). (2004). Hatun Willakuy. Versión abreviada del Informe Final. https:// idehpucp.pucp.edu.pe/wp-content/uploads/2012/11/hatun-willakuy-cvr-espanol.pdf

Crabtree, J. (2005). Alan García en el poder. Perú 1985-1990. Peisa.

Degregori, C. I. (2011). Qué difícil es ser Dios. El Partido Comunista del Perú - Sendero Luminoso y el conflicto armado interno en el Perú: 1980-1999. Instituto de Estudios Peruanos.

Domínguez, P., Burguette, E. y Bernard, A. (2003). Efectos del 11 de septiembre en la hotelería mexicana: reflexión sobre la monodependencia turística. Estudios y Perspectivas en Turismo, 12(3-4), 335-348.

Dorta, A. (2011). Crisis económica y cierre de establecimientos alojativos en destinos turísticos consolidados. El caso de Puerto de la Cruz (Tenerife). En Espacios y destinos turísticos en tiempos de globalización y crisis (Vol. II, pp. 183-197). AGE.

Drakos, K. y Kutan, A. M. (2003). Regional Effects of Terrorism on Tourism in Three Mediterranean Countries. Journal of Con ict Resolution, 47(5), 621-641.

Fernández, V. R. (2002). El Impacto del Terrorismo en las Llegadas de Turismo Internacional - Algunos Ejemplos. Anuario Turismo y Sociedad, 1, 70-79.

Fondo de Promoción Turística (FOPTUR). (1990). Anuario estadístico de turismo 1989. FOPTUR.

Fuller, N. (2008). Turismo y cultura. Entre el entusiasmo y el recelo. Pontificia Universidad Católica del Perú.

Gascón, J. (2005). Gringos como en sueños. Diferenciación y conflictos campesinos en los Andes peruanos ante el desarrollo del turismo. Instituto de Estudios Peruanos.

Gómez, L. T. (2016). Cómo afecta el factor peligrosidad en el momento que visita Colombia (Pro-manuscripto). TecnoCampus. Bogotá. 
Gómez, J. A. (2002). Turismo, conflicto armado y paz en Colombia: Apostándole al futuro. Anuario Turismo y Sociedad, 1, 57- 69.

Gonzáles, M. R. (2016). Impacto del terrorismo en el turismo [Tesis de grado, Universidad de Sevilla]. Sevilla.

Griffith, D. (1990). Turismo: Horizonte 2000. Anales. VI Congreso Nacional de Turismo, Cámara Nacional de Turismo (CANATUR), Lima.

Guilland, M. L. (2012). Colombia, el único riesgo es que te quieras quedar allí - De la promoción turística nacional al viaje a Sierra Nevada: usos y disuasión del riesgo. Via@, Imaginarios turiìsticos, 1. http://www.viatourismreview.net/Article4_ES.php

Heckmann, C. (1983, 1 de enero). El Comercio [p. C2].

Jaramillo, R. (2006). La hora del turismo. Ediciones Norma.

Korstanje, M. E. (2012). ¿Por qué a la industria turística le preocupa el terrorismo? Una introducción al problema. Anuario Turismo y Sociedad, (12), 147-167.

Korstanje, M. E. (2013). Preemption and Terrorism. When the Future Governs. Cultura. Revista Internacional de Filosofía de la Cultura y Axiología, 10(1), 167-184.

Korstanje, M. E. y Tarlow, P. (2012). Being lost: tourism, risk and vulnerability in the post-'9/11', entertainment industry. Journal of Tourism and Cultural Change, 10(1) 22- 33.

Loayza, S. (2018). Cusco y la industria del turismo durante el periodo de violencia. CCHIE 29-XI-2018. https://enfoquescusco. wordpress.com/2018/11/29/cusco-y-la-industria-del-turismo-durante-el-periodo-de-violencia/?fbclid=IwAR10LFIMtm GFF6PaMjbSJEnHNTWCeCbtHLuRXjCSxR76o5B0SX5jXTyUDiE

Marichal, C. (2010). Nueva historia de las grandes crisis financieras. Una perspectiva global, 1873-2008. Debate.

Maxwell, K. (2012). Tourism, Environment, and Development on the Inca Trail. Hispanic American Historical Review, 92(1), 143171.

Ministerio de Industria, Comercio, Turismo e Integración (MICTI). (1985). Memoria de la gestión en el sector turismo del señor ministro Ing. Álvaro Becerra Sotero correspondiente al período abril 1984 - julio 1985. MICTI.

Ministerio de Industria, Turismo, Integración y Negociaciones Comerciales Internacionales (MITINCI). (1993). Compendio Estadístico del sector Turismo 1970-1992 [2 tomos]. Lima: MITINCI.

Monti, E. (2011). La crisis económica internacional de 2008 y el turismo: efectos y medidas de respuesta en Rio Grande do Norte, Brasil. Investigaciones Turísticas, 1, 93-106.

Moral, P. (2016). El impacto del terrorismo internacional en la industria del turismo: balance y perspectivas en el Mediterráneo. Instituto Español de Estudios Estratégicos.

Morales, R. (2009). El impacto de la crisis económica mundial sobre la economía costarricense: los efectos macroeconómicos. Economía y Sociedad, 14(35-36), 61-73.

Moreno-Bird, J. C., Pérez, E. y Ruiz, P. (2004-2005). The Washington Consensus: A Latin American Perspectives Fifteen Years Later. Journal of Post Keynesian Economics, 27(2), 345-365.

Murakami, Y. (2007). Perú en la era del «Chino». La política no institucional y el pueblo en busca de un salvador. Instituto de Estudios Peruanos y Center for Integrated Area Studies, Kyoto University.

Observatorio Turístico del Perú (OTP). (2018). BADATUR. Banco de datos turísticos del Perú. Información económica, financiera y turística. Universidad de San Martín de Porres. http://www.observatorioturisticodelperu.com/badatur/info-peru/datos-generales

O’Hare, G. y Barret, H. (1997). The Destination Life Cycle: International Tourism in Peru. Scotttish Geographical Magazones, 113(2), 66-73.

Organización Mundial del Turismo (OMT). (1995). Compendio de Estadísticas de Turismo 1989-1993. OMT.

Parodi, C. (2005). Perú 1960-2000. Políticas económicas y sociales en entornos cambiantes. Universidad del Pacífico.

Rodríguez, O. E. (1984). El desarrollo del sector turismo y su incidencia en la economía nacional, 1963-1980 [Tesis de grado en economía, Universidad de Lima]. Lima. 
Saha, S. y Yap, G. (2013). The moderation effects of political instability and terrorism on tourism development: a cross-country panel analysis. Journal of Travel Research, 53(4), 509-521.

Sector turismo y su privatización en el Perú. (1994). Efficacia, 10(10), 31-32.

Terrorismo, la imagen y el turismo. (1989, 13 de noviembre). El Comercio.

Torres, E. (2014). La crisis económica en el sector turístico. Un análisis de sus efectos en la costa del sol. Análisis Turístico, 18 , 11-18.

Valle-Cornavaca, A. L. (2015). Antecedentes históricos del turismo y su evolución en Nicaragua. Revista de Investigaciones Sociales, 1(1), 36-49.

Valle, M. E. (1991, 15 de julio). El cólera y el terrorismo dañan turismo en Nazca. El Comercio.

Van Broeck, A. M. (2002). Turismo doméstico en Colombia en un contexto de conflicto armado (1996 - 2001). Anuario Turismo y Sociedad, 1, 44-56.

Wall, G. (1996). Terrorism and Terrorism: An Over- view and an Irish Example. In A. Pizam \& Y. Mansfeld (Eds.), Tourism, Crime and International Security Issues. John Willey \& sons.

Williamson, J. (2004). A Short History of the Washington Conference [Paper for a conference]. Fundación CIDOB, From the Washington Consensus Towards a New Global Governance.

Wise, C. (2010). Reinventando el Estado: estrategia económica y cambio institucional en el Perú. Universidad del Pacífico.

\section{Fernando Armas Asín}

Historiador, doctor en su disciplina por la Universidad de Navarra (España). Actualmente es profesor en la Universidad del Pacífico (Perú). Tiene múltiples libros y artículos dedicados a la historia económica, con énfasis en los campos del turismo y de la religión. Fue investigador externo de la Universidad de San Martín de Porres (Perú) y es miembro de la Academia Nacional de la Historia y de la Asociación Peruana de Historia Económica.

armas_fa@up.du.pe

ORCID: https://orcid.org/0000-0001-7050-1423 